\title{
Evaluación del riesgo de explosividad del gas metano en minería subterránea de carbón, caso de la cuenca del Sinifaná, Colombia
}

\author{
Camilo Andrés Ortega-Ramos ${ }^{1 *}$; Triana Margarita Franco-Bonfante ${ }^{1}$; \\ Astrid Blandón-Montes ${ }^{1}$; Jorge Martín Molina-Escobar ${ }^{1}$
}

DOI: http://dx.doi.org/10.18273/revbol.v40n1-2018005 @c) (1)

Forma de citar: Ortega-Ramos, C.A., Franco-Bonfante, T.M., Blandón-Montes, A., y Molina-Escobar, J.M. (2018). Evaluación del riesgo de explosividad del gas metano en minería subterránea de carbón, caso de la cuenca del Sinifaná, Colombia. Boletín de Geología, 40(1), 83-91. Doi: 10.18273/revbol.v40n1-2018005.

\section{RESUMEN}

El metano asociado al carbón es un gas que potencialmente genera riesgos de explosión en labores de minería subterránea. Este gas es explosivo cuando se encuentra dentro de un rango del volumen entre 5\% y $15 \%$, por lo que es de gran importancia realizar monitoreos constantes de las concentraciones. El punto más crítico de la actividad minera es el arranque de carbón ya que es el momento donde se hace una mayor perturbación de este ocasionando la liberación del gas. Se realizaron mediciones de concentraciones de metano durante el arranque en minas a lo largo de la cuenca del Sinifaná, a partir de las cuales se realizaron perfiles y correlaciones para determinar los factores más influyentes para las acumulaciones de gas y por ende explosiones, además se muestran correlaciones entre factores como la ventilación y las ubicaciones geográficas de las minas siendo las primeras las más influyentes al presentarse concentraciones de un 30\% más altas en minas sin ventilación.

Palabras clave: metano; arranque; carbón; Sinifaná.

\section{Explosibility risk evaluation of the methane gas in coal underground mining, case Sinifaná basin, Colombia}

\begin{abstract}
Coal-Bed Methane (CBM) has the risk to generate explosions when its volume is between 5\% and $15 \%$, so it is very important to perform a routine monitoring of its concentrations during mining activities. The most critical point of the coal mining is the digging because it is the moment when the perturbation of CBM occurs, causing its release. Were performed measurements of methane concentrations during the digging in mines along the Sinifaná basin, from wich were performed profiles and correlations to determine the most influential factors for gas accumulations and explosions. Also, they were performed correlations between factors of ventilation and geographical locations of the mines, being the first the most influential it presenting concentrations of $30 \%$ higher in mines without ventilation.
\end{abstract}

Keywords: methane; digging; coal; Sinifaná.

1 Facultad de Minas, Universidad Nacional de Colombia, Medellín, Colombia. (*)caaortegara@unal.edu.co, tmfrancob@unal.edu.co, asblando@unal.edu.co,jmmolina@unal.edu.co 


\section{INTRODUCCIÓN}

La minería subterránea de carbón es una labor donde se tiene el riesgo latente de las explosiones por atmosferas con altas concentraciones de gas metano $\left(\mathrm{CH}_{4}\right)$ producido por el carbón, por lo tanto, conocer el gas desprendido en los procesos de extracción es de gran utilidad para saber de manera precisa las condiciones a las que están sometidos los trabajadores. Es posible evaluar de distintas formas la cantidad de metano que se está generando en un frente de explotación durante las labores de arranque, perforación y voladura en el carbón.

Las emisiones de gas durante estas labores se han podido determinar tanto por métodos experimentales como por modelamientos numéricos. Esto se realiza debido a que la cantidad de metano presente en el frente de un túnel es diferente a la que se encuentra en el manto in-situ, es decir, el conocimiento de esto nos puede ayudar para saber la cantidad real de metano que se tiene presente y que se debe diluir (Karacan et al., 2011).

En el proceso minero, el arranque podría ser el punto más crítico porque es el momento en que mayor metano se desprende, ya que puede afectar las variables que controlan la cantidad de metano desprendido, debido a esto se hacen muy importantes estudios donde se muestran los efectos causados por el arranque sobre los mantos (ALL Consulting, 2003).

La cuenca del Sinifaná cuenta con un alto riesgo de presenciar explosiones de metano, debido a que en la mayor parte de las minas no se realizan mediciones de gas durante los procesos de arranque, lo que incrementa la posibilidad de ocurrencia de estas.

\section{MARCO TEÓRICO}

\section{Ubicación de la zona de estudio}

La cuenca del Sinifaná se encuentra ubicada al Suroeste de la ciudad de Medellín y se encuentra conformada por 5 municipios todos pertenecientes al departamento de Antioquia: Amaga, Venecia, Titiribí, Angelópolis y Fredonia. En todos, la minería subterránea de carbón es una de las actividades económicas principales y gran parte de esta se considera pequeña minería. La FIGURA 1 muestra la región que abarca la cuenca del Sinifaná.

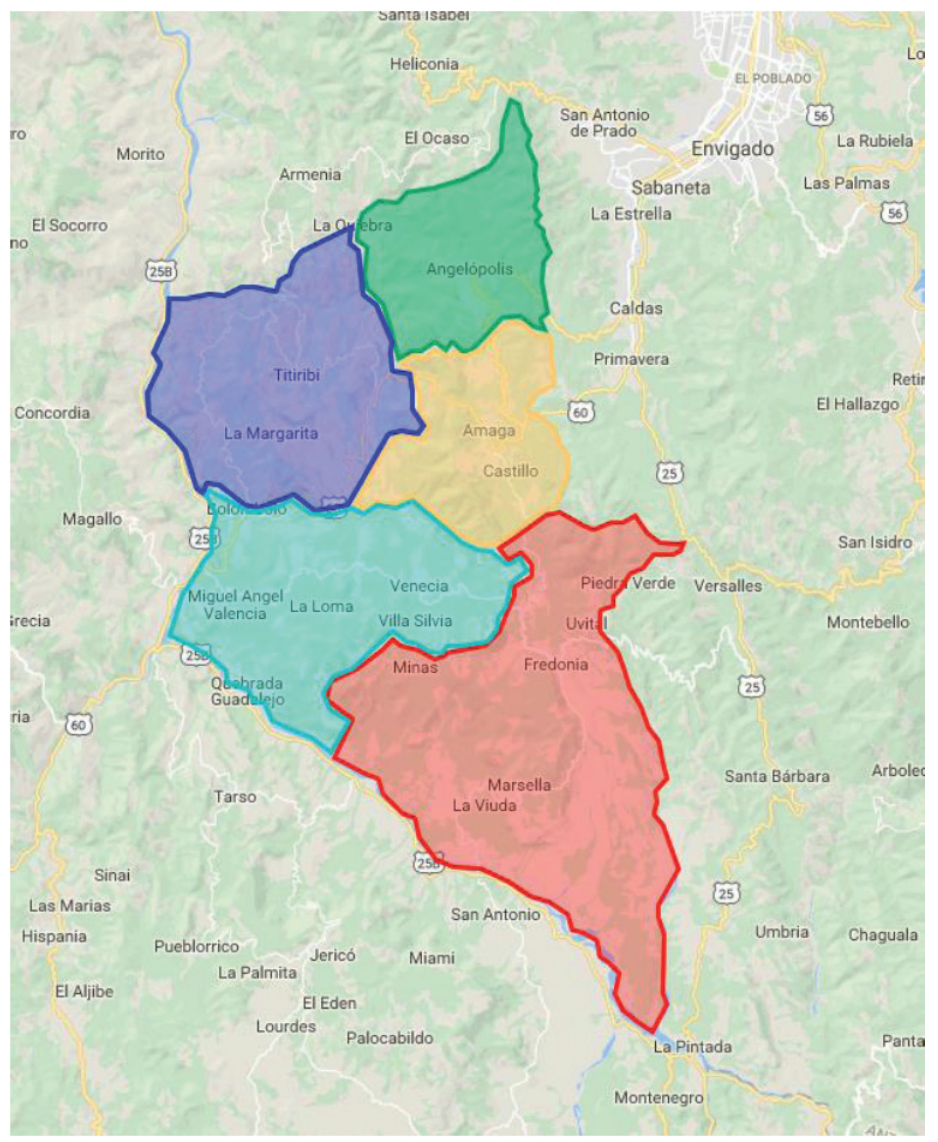

FIGURA 1. Cuenca del Sinifaná, Antioquia. Modificado de Google Maps, 2017. 


\section{Gas asociado al carbón}

El gas asociado al carbón o CBM (Coal Bed Methane) es un hidrocarburo no convencional fundamentalmente diferente en su proceso de acumulación y tecnología de producción; es un gas natural que contiene 100\% del metano proveniente de los reservorios del manto de carbón y con frecuencia se produce a profundidades superficiales y asociado a grandes volúmenes de agua de calidad variable (ALL Consulting, 2003; Anna, 2003; Moore, 2012).

El CBM es gas natural que se genera y almacena en un manto de carbón. La producción de este se realiza por medio de un pozo de tal modo que el gas y el agua se llevan a la superficie (ALL Consulting, 2003; Anna, 2003; Moore, 2012).

Los recursos de CBM representan volúmenes comerciales de gas natural dentro y fuera de áreas de producción convencional de aceite y gas (ALL Consulting, 2003; Flores et al., 2008; Hackley et al., 2007; Islam y Hayashi, 2008; Shi et al., 2002).

El CBM es metano con pequeñas cantidades de otros gases de hidrocarburos y no hidrocarburos que se presenta naturalmente en los mantos de carbón como resultado de los procesos químicos y físicos (ALL Consulting, 2003; Moore, 2012).

Con el aumento del enterramiento y la maduración, los compuestos orgánicos liberan agua, $\mathrm{CO}_{2}$, metano y otros gases. Físicamente, el material pierde porosidad debido a los cambios en compactación y maduración. La reducción en la porosidad sucede debido a la compactación y deformación de los macerales del carbón (ALL Consulting, 2003; Moore, 2012).

A medida que el carbón madura a tipos bituminosos, el agua se expele, la porosidad decrece y la formación de metano biogénico decrece debido al ascenso de la temperatura por encima del rango más favorable para las arqueas (Thauer y Shima, 2006). Al mismo tiempo, el calor descompone los compuestos orgánicos complejos para desprender metano e hidrocarburos más pesados (etano y más altos) (ALL Consulting, 2003; Moore, 2012).

A medida que la vitrinita se somete a maduración, su contenido de carbono aumenta, y el de volátiles disminuye, se vuelve más duro y brillante, su reflectancia incrementa en la medida que madura. La reflectancia de la vitrinita es una medida de la reflectividad del carbón y de esta manera se puede determinar el rango del carbón (ALL Consulting, 2003; Moore, 2012).

El gas asociado al carbón (CBM) existe en el carbón en tres estados básicos: como gas libre, como gas disuelto en agua en el carbón y como gas adsorbido sobre la superficie sólida del carbón (ALL Consulting, 2003; Moore, 2012).

Con el fin de que los fluidos salgan de los mantos de carbón en un pozo, el carbón debe poseer un sistema de permeabilidad secundaria tal como fracturas, estas permiten que el agua, el gas natural y otros fluidos migren desde la porosidad de la matriz hacia el pozo en producción (ALL Consulting, 2003; Laubach et al., 1998). Diaclasas es el término para la red de fracturas naturales que se forman en los mantos de carbón como parte de la maduración del carbón (ALL Consulting, 2003; Laubach et al., 1998).

En los mantos de carbón, mucho gas es absorbido sobre las laminaciones microscópicas y microporos dentro de los macerales del carbón (ALL Consulting, 2003).

A medida que la presión hidrostática disminuye por la producción de agua, el gas se desorbe y se mueve dentro del sistema de diaclasas donde este empieza a fluir hacia el pozo en producción. El gas natural también puede migrar a través del conjunto de fracturas más amplias relacionadas a fallas y diaclasamiento (ALL Consulting, 2003; Laubach et al., 1998).

\section{Atmosferas explosivas}

Las atmosferas se consideran explosivas por la existencia en el aire de sustancias inflamables que en contacto con una fuente de ignición provoca una explosión (Instituto Nacional de Seguridad e Higiene en el Trabajo, 2003).

Para las minas de carbón, es importante conocer que las labores se realizan en condiciones propensas a que se generen explosiones. Por esto las instalaciones en las minas deben cumplir normas para sus equipos y para las formas de trabajo las cuales no deben ser susceptibles a explosiones (Fernández et al., 2003).

Para que la mezcla de aire y metano se le pueda dar ignición se requiere que se presenten ciertas condiciones, tales como: la concentración de metano, el volumen de la mezcla y la uniformidad de esta misma. La mezcla que genera la explosión más violenta se da cuando la concentración es de 9,5\% y se requiere como 
mínimo $4 \mathrm{~m}^{3}$ de mezcla para que esta sea capaz de iniciarse, es decir aproximadamente $0,4 \mathrm{~m}^{3}$ de metano (Charris, 2013).

En términos del agente de ignición, las explosiones pueden iniciarse por distintas fuentes, como lo son las llamas abiertas, cortos circuitos, circuitos eléctricos no sellados intrínsecamente, descargas eléctricas y electricidad estática (Clavijo, 2016).

Para una evaluación de las condiciones en las minas subterráneas se pueden seguir las normas para el control de atmosferas explosivas, son útiles las Normas ATEX-100 que basa su normativa en aparatos y sistemas de protección para uso en atmósferas explosivas; la ATEX-137, basada en la protección de la seguridad y la salud de los trabajadores expuestos a los riesgos derivados de atmosferas explosivas en lugares de trabajo (Fernández et al., 2003). Y la normativa NFPA 122, para la prevención y control de incendios en minería metálica/no metálica.

\section{METODOLOGÍA EXPERIMENTAL}

Para evaluar el riesgo de explosión al cual están sometidas las minas de la cuenca del Sinifaná se efectuaron mediciones de concentraciones de metano en los frentes de explotación de distintas minas durante los procesos de arranque.

\section{Equipos utilizados}

Para las mediciones de concentraciones se utilizaron los sensores infrarrojos para metano Crowcon Detective+. La FIGURA 2 muestra el equipo utilizado para los ensayos.

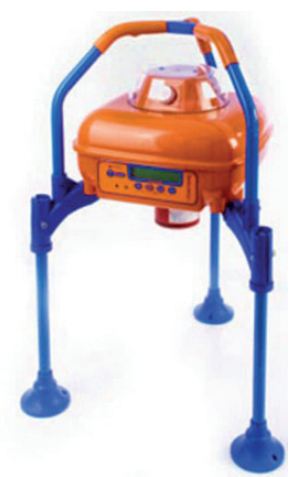

FIGURA 2. Sensor infrarrojo de metano Crowcon Detective+ . Tomado de Crowcon (2017).

La TABLA 1 muestra las características del equipo utilizado. Además de esto, el equipo cuenta con una manguera y una bomba de succión lo que permite realizar medidas de concentraciones de gases en lugares donde no puede acceder el equipo.

TABLA 1. Gases y rango de mediciones ofrecida por el sensor. Fuente: Crowcon (2017).

\begin{tabular}{ll}
\hline \multicolumn{1}{c}{ Gas } & \multicolumn{1}{c}{ Rango de medición } \\
\hline Metano $\left(\mathrm{CH}_{4}\right)$ & $0-100$ LEL (Límite \\
& inferior de explosividad) \\
Metano $\left(\mathrm{CH}_{4}\right)$ & $5-100 \%$ Volumen \\
Monóxido de carbono $(\mathrm{CO})$ & $0-500 \mathrm{ppm}$ \\
Oxigeno $\left(\mathrm{O}_{2}\right)$ & $0-25 \%$ Volumen \\
\hline
\end{tabular}

\section{Ensayos realizados}

Ensayo para el arranque manual: El objetivo de este ensayo es la determinación de las concentraciones máximas de metano alcanzadas en el frente de explotación y el perfil de estas en el túnel cuando se realiza arranque del carbón por martillo neumático o pica manual.

El montaje del experimento consiste en la ubicación de los sensores a lo largo del túnel como se muestra en la FIGURA 3A, apoyándose de las mangueras de los sensores la medición de datos se ejecutó a una altura de la mitad del alto del túnel. Se realizó una toma de datos por 10 minutos, después de esto los últimos 3 sensores se movieron a más distancia del frente con el fin de obtener más puntos de medición, la toma de datos se mantuvo nuevamente por 10 minutos (FIGURA 3B). El primer sensor permaneció en el mismo sitio con el fin de obtener la medición de concentración en el frente que es el lugar donde hay la mayor concentración.

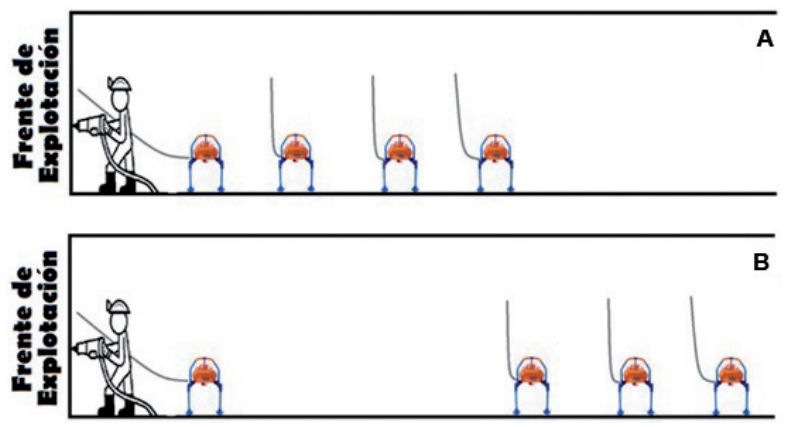

FIGURA 3. A. Ensayo de medición de concentraciones en el arranque manual, momento inicial. B. Ensayo de medición de concentraciones en el arranque manual, momento final.

Ensayo para el arranque por voladura: El objetivo de este ensayo es la determinación de las concentraciones máximas de metano alcanzadas en el frente de 
explotación y el perfil de estas en el túnel cuando se realiza arranque del carbón por medio de una voladura.

El experimento de medición del comportamiento de gases durante el arranque por voladura requiere la ubicación de los sensores a lo largo del túnel. La boca de la manguera de los sensores se ubicó a una altura de la mitad del alto del túnel ya que la emisión de metano por la voladura se da una sola vez, la ubicación de los sensores no se cambió. La manguera del primer sensor se ubicó en el frente para obtener la primera emisión. La FIGURA 4 muestra el esquema de las ubicaciones de los sensores para este caso.

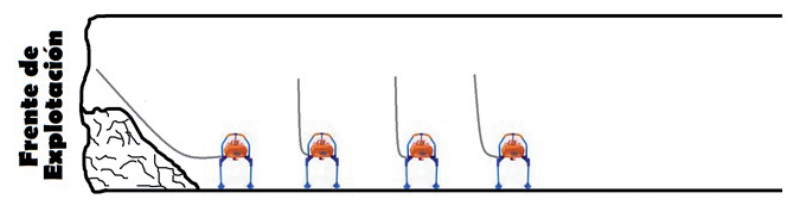

FIGURA 4. Ensayo de medición de concentraciones en el arranque por voladura.

\section{RESULTADOS Y DISCUSIÓN}

Para los ensayos mostrados en la sección de Metodología Experimental se visitaron un total de 14 minas en la región de la cuenca del Sinifaná. La TABLA 2 muestra las minas visitadas con su ubicación y el tipo de ensayo realizado según el método de explotación utilizado.

TABLA 2. Minas visitadas con su ubicación y el tipo de ensayo realizado según el método de explotación utilizado.

\begin{tabular}{cll}
\hline Mina & \multicolumn{1}{c}{ Ubicación } & \multicolumn{1}{c}{ Tipo de ensayo } \\
\hline NE & Amagá & Arranque por voladura \\
TC & Angelópolis & Arranque mecánico \\
SF & Amagá & Arranque por voladura \\
BE & Angelópolis & Arranque mecánico \\
LM & Titiribí & Arranque mecánico \\
DG & Angelópolis & Arranque mecánico \\
JP & Angelópolis & Arranque mecánico \\
SP & Venecia & Arranque mecánico \\
LB & Angelópolis & Arranque mecánico \\
M & Angelópolis & Arranque mecánico \\
LH & Venecia & Arranque mecánico \\
ME & Amagá & Arranque mecánico \\
A & Fredonia & Arranque por voladura \\
EB & Fredonia & Arranque por voladura \\
\hline
\end{tabular}

Las FIGURAS 5, 6 y 7 muestran los perfiles de concentración para las minas donde se realizaron ensayos de arranque manual con pica, martillo picador y voladura respectivamente obtenidos de los ensayos en cada una de las minas, en estas se muestran los perfiles de las minas con las menores concentraciones y las mayores.

A partir de los resultados obtenidos se puede buscar una correlación entre el método de arranque y la emisión de metano de un manto, ya que de acuerdo a la forma en que se perturbe el carbón se pueden obtener cambios en la variable de permeabilidad, la cual influye directamente en la tasa de emisión de metano, al facilitar la movilidad de este a través de las discontinuidades del carbón.

En las FIGURAS 5, 6 y 7, se pueden observar concentraciones máximas similares menores a 9 LEL en algunas de las minas de los municipios de Angelópolis, Amagá y Fredonia, a pesar de utilizar distintos métodos de arranque (pica, martillo neumático y voladuras), a excepción del caso particular de una mina del municipio de Titiribí la cual presenta una concentración superior a las demás, incluso a las que explotan por su mismo método, tomando en base estos patrones se puede decir que para el caso en particular de la cuenca del Sinifaná el método de arranque no es un factor fuertemente influyente en las concentraciones de metano en las minas. La FIGURA 10 muestra la concentración promedio de metano en el ambiente mientras se realizaba el arranque de carbón para cada mina.

Además del método de arranque existen otros factores que influyen en las concentraciones de metano obtenidas en los resultados, por ejemplo, la ventilación. En la cuenca del Sinifaná de las 14 minas visitadas 4 aun cuentan con ventilación por tiro natural. En las minas con ventilación mecánica se evidenciaron concentraciones promedio menores que las que contaban con ventilación totalmente por tiro natural como se evidencia en la FIGURA 8.

Las concentraciones en minas que ventilan sus frentes con ventilación natural son mayores debido a que el caudal que ingresa no es suficiente para que las emisiones de metano que se producen durante el arranque del carbón se diluyan. En estos casos de ventilación por tiro natural se logró evidenciar en algunas de las minas concentraciones mayores a $20 \mathrm{LEL}$, es decir, mayores a $1 \%$ en volumen de Metano. 

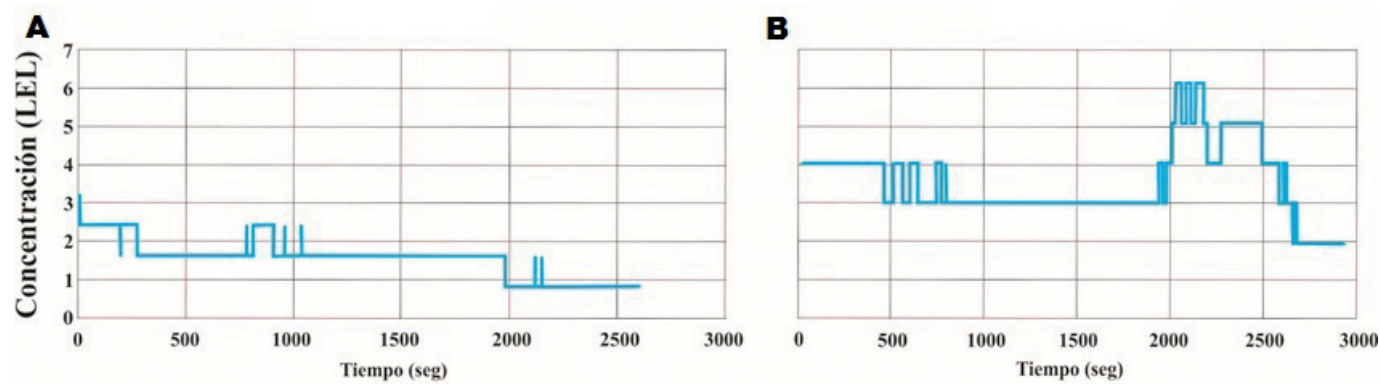

FIGURA 5. A. Perfil de concentraciones durante el arranque en mina JP (Extracción por pica y pala). B. Perfil de concentraciones durante el arranque en mina M (Extracción por pica y pala).
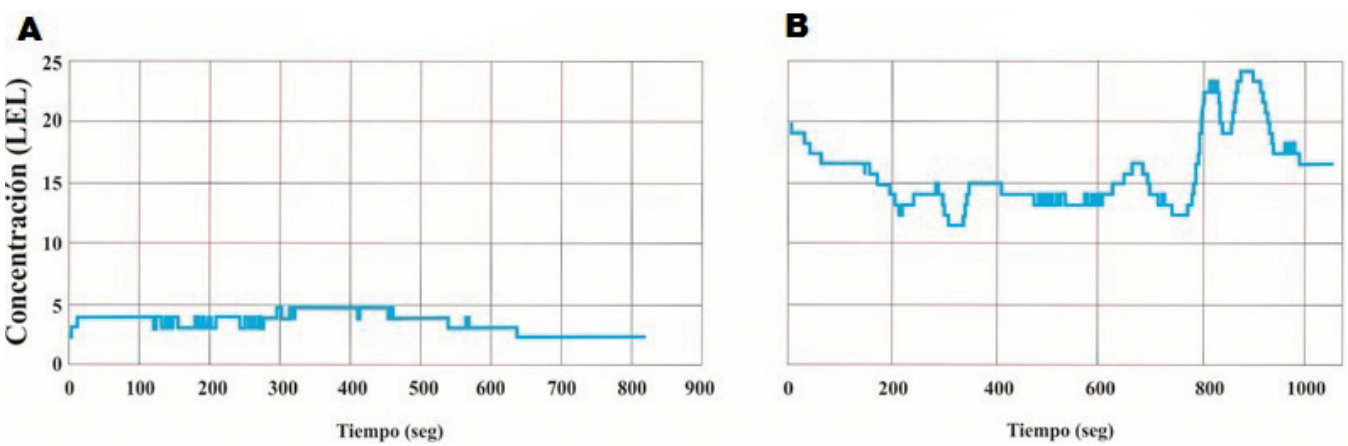

FIGURA 6. A. Perfil de concentraciones durante el arranque en mina CM (Extracción por martillo neumático). B. Perfil de concentraciones durante el arranque en mina LM (Extracción por martillo neumático).

A

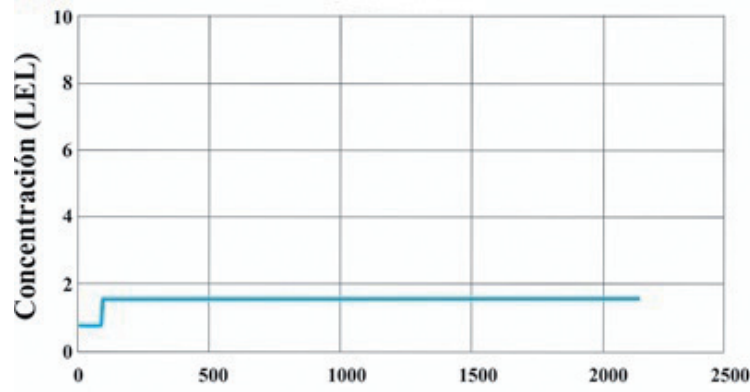

B

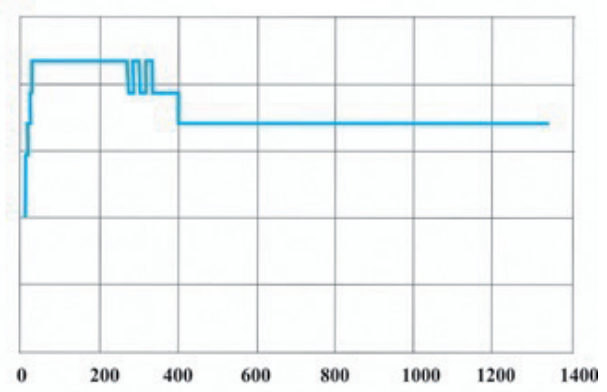

FIGURA 7. A. Perfil de concentraciones durante el arranque en mina SF (Extracción por voladura). B. Perfil de concentraciones durante el arranque en mina A (Extracción por voladura).

En vista de que las condiciones del carbón pueden variar de acuerdo a la ubicación de este, es importante también analizar las concentraciones promedio por municipio. Conforme a esto la FIGURA 9 muestra las concentraciones promedio por municipio, en la cual se notó que las concentraciones más altas se encuentran en minas del Municipio de Titiribí. Este resultado va de la mano con la correlación anterior por ventilación, ya que la mina en la que se obtuvo mayores concentraciones debido a su sistema de ventilación natural se encuentra en este municipio, mientras que en los demás municipios se encuentran en un rango similar entre ellos (menores a 6 LEL).
Por los análisis y resultados obtenidos es posible decir que el factor más influyente por el cual se presentan condiciones peligrosas de acumulación de metano es la ventilación, las velocidades bajas del aire favorecen la estratificación y acumulación de este en algunos puntos. Otro factor que incrementa el riesgo de concentraciones altas de gas son las llamadas bolsas de metano, que normalmente son zonas asociadas a fallas inversas (Seidle, 2011), en las cuales este se acumula y son encontradas en el avance de los trabajos mineros, liberando todo el gas en poco tiempo, haciendo que la ventilación que se utiliza en dicho momento no sea suficiente y por lo tanto, se pueden presentar fácilmente accidentes fatales. 


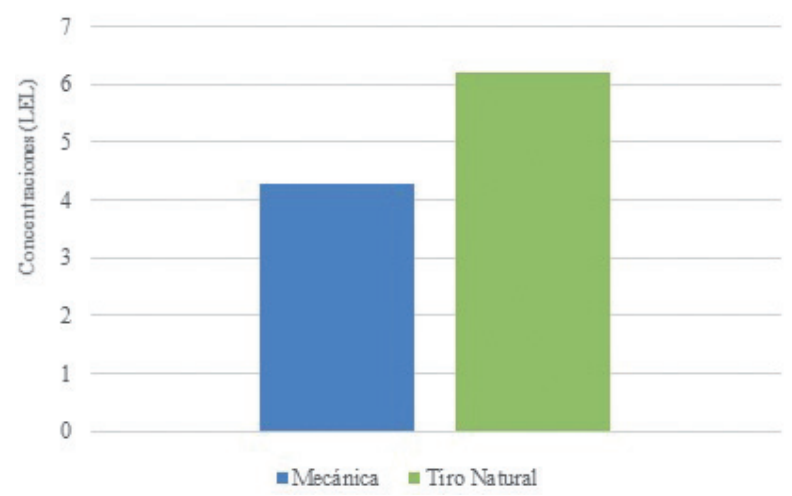

FIGURA 8. Concentraciones de metano según el tipo de ventilación.

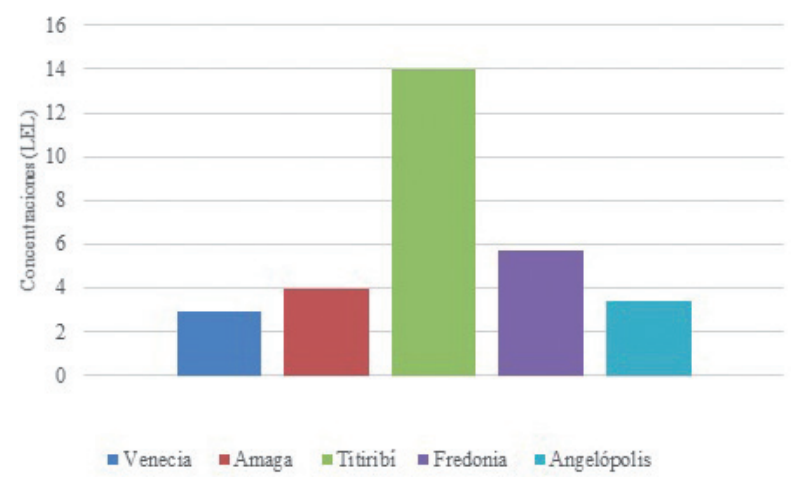

FIGURA 9. Concentraciones promedio de metano por municipio.

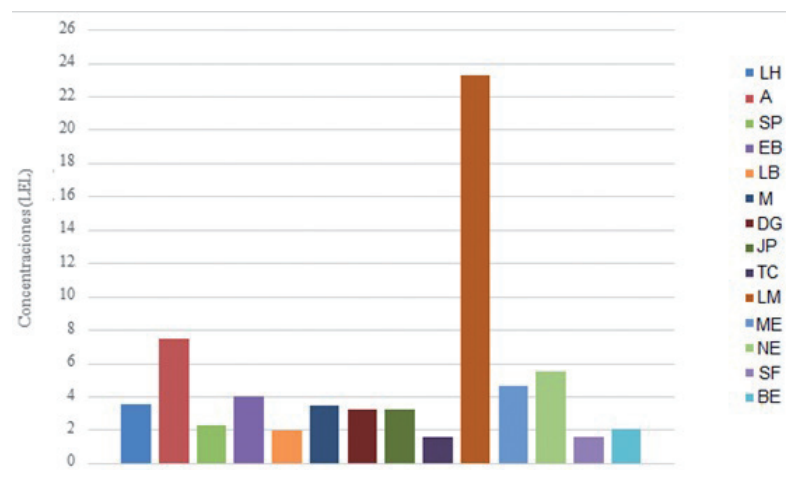

FIGURA 10. Concentraciones promedio de metano durante el arranque en las minas visitadas.

Para el cálculo cuantitativo de las emisiones de metano en los frentes se partió del uso de las concentraciones promedios durante los ensayos y los caudales de aire en los sitios de explotación que fueron medidos con un anemómetro digital. La ecuación 1 muestra como calcular el caudal de emisiones de metano durante el arranque de carbón.

$$
Q_{C H 4}=Q_{a} C_{C H 4}
$$

Donde, $\mathrm{Q}_{\mathrm{CH} 4}$ es el caudal de emisiones de metano en $\mathrm{m}^{3} / \mathrm{s}, \mathrm{Q}_{\mathrm{a}}$ es el caudal de aire medido en $\mathrm{m}^{3} / \mathrm{s} \mathrm{y}_{\mathrm{CH} 4}$ es la concentración de metano promedio en el ambiente en LEL.

La ecuación 1 se debe multiplicar por un factor para realizar la conversión de unidades, esta conversión es $5 \% \mathrm{Vol}$ corresponde a $100 \mathrm{LEL}$.

Sabiendo esto, la TABLA 3 muestra las minas donde se realizaron los ensayos con sus respectivos caudales medidos, concentraciones promedios y emisiones de metano calculadas. Adicionalmente las minas donde el anemómetro no era capaz de medir la velocidad del aire en el túnel debido a que era muy baja se les dio un valor de "menor a $0,5 \mathrm{~m} / \mathrm{s}$ " que es el rango de incertidumbre del equipo utilizado, en algunos casos fue posible medir caudales menores gracias a que se poseían ductos de ventilación donde si era posible medir una velocidad al aire.

TABLA 3. Minas con sus caudales de aire, concentraciones promedio de metano y sus emisiones de gas.

\begin{tabular}{cc|c|c}
\hline Mina & $\mathbf{Q}_{\mathbf{a}}\left(\mathbf{m}^{3} / \mathbf{s}\right)$ & $\mathbf{C}_{\mathrm{CH} 4}(\mathbf{L E L})$ & $\mathbf{Q}_{\mathrm{CH} 4}\left(\mathbf{m}^{3} / \mathbf{s}\right)$ \\
\hline $\mathrm{NE}$ & 1,10 & 5,50 & 0,00302 \\
$\mathrm{TC}$ & $<0,50$ & 1,60 & $<0,0004$ \\
$\mathrm{SF}$ & 2,01 & 1,55 & 0,00402 \\
$\mathrm{BE}$ & $<0,50$ & 2,10 & $<0,00052$ \\
$\mathrm{DG}$ & $<0,50$ & 3,26 & $<0,00081$ \\
$\mathrm{JP}$ & $<0,50$ & 3,26 & $<0,00081$ \\
$\mathrm{LB}$ & $<0,50$ & 2,00 & $<0,0005$ \\
$\mathrm{M}$ & 0,68 & 3,47 & 0,00119 \\
$\mathrm{LH}$ & 0,14 & 3,99 & 0,00029 \\
$\mathrm{LM}$ & $<0,50$ & 4,66 & $<0,00116$ \\
$\mathrm{~A}$ & 0,19 & 7,46 & 0,00074 \\
$\mathrm{~EB}$ & 0,35 & 3,63 & 0,00063 \\
\hline
\end{tabular}

En la TABLA 3 se puede observar que las emisiones de metano en el tiempo muestran valores bastante bajos siendo el mayor de $0,00402 \mathrm{~m}^{3} / \mathrm{s}$, lo que también apunta a que el riesgo más latente son las acumulaciones del gas en los puntos críticos, es decir donde no hay ventilación.

\section{CONCLUSIONES}

- Durante las visitas realizadas en minas a lo largo de la cuenca del Sinifaná, no se evidenciaron concentraciones que representen riesgos de explosiones por metano, por lo que es posible decir 
que los eventos de este tipo ocurridos en la cuenca corresponden a situaciones particulares donde las concentraciones se incrementan rápidamente hasta el rango de explosividad, como lo son el caso de las acumulaciones en puntos críticos o las bolsas de metano.

- Debido a las características geológicas del carbón, las concentraciones registradas de metano en las minas son muy bajas, así, para evitar el riesgo de explosiones se debe enfocar en controlar las acumulaciones de gas por medio de ventilación mecánica y garantizando el régimen turbulento en los túneles para que el metano no se estratifique en el techo.

- Hay factores más influyentes que otros en la creación de atmosferas explosivas, como lo es el caso de la profundidad a la cual se encuentran los frentes de explotación, la ventilación principal que en algunos casos es por tiro natural, encuentra mucha resistencia aerodinámica en los trabajos muy alejados, llegando poco caudal a estas zonas. En este tipo de casos es necesaria ventilación auxiliar para evitar concentraciones altas de metano.

- Cuantitativamente se obtuvieron emisiones de metano constantes que no representan un riesgo a la seguridad ya que son muy bajas, aunque cabe que en el trascurso del tiempo este podría acumularse en sitios sin ventilación.

\section{AGRADECIMIENTOS}

Los autores expresan su profundo agradecimiento a las empresas mineras y pequeños mineros que permitieron realizar mediciones y pruebas en sus instalaciones, por permitir y promover estas investigaciones.

\section{REFERENCIAS}

ALL Consulting. (2003). Handbook on coal bed methane produced water: Management and beneficial use alternatives. Chapter 2. Tulsa, Oklahoma: ALL Consulting.

Anna, L.O. (2003). Groundwater flow associated with coalbed gas production, Ferron Sandstone, east-central Utah. International Journal of Coal Geology, 56(1-2), 69-95. doi: 10.1016/S01665162(03)00077-6.

ATEX 100. Directiva de aparatos y sistemas de protección contra atmósferas explosivas. 1996.
ATEX 137. Protección de la seguridad y la salud de los trabajadores expuestos a los riesgos derivados de la presencia de atmósferas explosivas en el lugar de trabajo. 1999.

Charris, T. (2013). Resumen de ideas en la aplicación de la ingeniería total en una mina de carbón, con protocolos y normas para minas de carbón, en especial minas grisúgenas. Consultado el 2 de febrero de $2017 . \quad$ http://www.fedecundi.com/ empresarioseingenieros.pdf.

Clavijo, J.A. (2016). Monitoreo de gases en minería. Instrumentación especializada higiene y seguridad. Agencia Nacional de Minería. Consultado el 16 de noviembre de 2016. http://www.anm.gov.co/ sites/default/files/DocumentosAnm/presentacion multidetectores_ibrid-mx6.pdf.

Crowcon. (2017). Crowcon Detective +. Consultado el 28 febrero 2017. https://www.crowcon.com/uk/ products/portables/detective-plus.html.

Fernández, C., García, J., y Vega, A. (2003). Breve guía sobre productos e instalaciones en atmosferas explosivas. Madrid: Laboratorio oficial J.M. Madariaga.

Flores, R.M., Rice, C.A., Stricker, G.D., Warden, A., and Ellis, M.S. (2008). Methanogenic pathways of coal-bed gas in the Powder River Basin, United States: The Geologic factor. International Journal of Coal Geology, 76(1-2), 52-75. doi: 10.1016/j. coal.2008.02.005.

Hackley, P.C., Warwick, P.D., and Breland Jr, F.C. (2007). Organic petrology and coalbed gas content, Wilcox Group (Paleocene-Eocene), Northern Louisiana. International Journal of Coal Geology, 71(1), 54-71. doi: 10.1016/j. coal.2006.05.009.

Instituto Nacional de Seguridad e Higiene en el Trabajo (2003). Atmósferas explosivas en el lugar de trabajo. España. Consultado el 16 de noviembre de 2016. http://www.insht.es/InshtWeb/ Contenidos/Normativa/GuiasTecnicas/Ficheros/ ATM\%C3\%93SFERAS\%20EXPLOSIVAS.pdf.

Islam, M.R., and Hayashi, D. (2008). Geology and coal bed methane resource potential of the Gondwana Barapukuria coal basin, Dinajpur, Bangladesh. International Journal of Coal Geology, 75(3), 127-143. 
Karacan, C.Ö., Ruiz, F.A., Cotè, M., and Phipps, S. (2011). Coal mine methane: A review of capture and utilization practices with benefits to mining safety and to greenhouse gas reduction. International Journal of Coal Geology, 86(2-3), 121-156. doi: 10.1016/j.coal.2011.02.009.

Laubach, S.E., Marrett, R.A., Olson, J.E., and Scott, A.R. (1998). Characteristics and origins of coal cleat: A review. International Journal of Coal Geology, 35(1-4), 175-207. doi: 10.1016/S01665162(97)00012-8.

Moore, T.A. (2012). Coalbed methane: A review. International Journal of Coal Geology, 101, 3681. doi: 10.1016/j.coal.2012.05.011.

NFPA 122. (2015). Standard for Fire Prevention and Control in Metal/Nonmetal Mining and Metal Mineral Processing Facilities. National Fire Protection Association.

Seidle, J. (2011). Fundamentals of coalbed methane reservoir engineering. Chap. 3. Geologic aspects of coal gas reservoir engineering (pp. 59-88). Oklahoma: PennWell Books.

Shi, J.Q., Durucan, S., and Sinka, I.C. (2002). Key parameters controlling coalbed methane cavity well performance. International Journal of Coal Geology, 49(1), 19-31. doi: 10.1016/S01665162(01)00054-4.

Thauer, R.K., and Shima, S. (2006). Biogeochemistry: Methane and microbes. Nature, $\quad 440(7086), \quad 878-879 . \quad$ doi: $10.1038 / 440878 \mathrm{a}$.

Camilo Andrés Ortega-Ramos. ORCID: 0000-0003-4047-2195

Triana Margarita Franco-Bonfante.

ORCID: 0000-0002-5882-1552

Astrid Blandón-Montes. ORCID: 0000-0003-4537-2046

Jorge Martín Molina-Escobar. ORCID: 0000-0002-6068-2571

Trabajo recibido: mayo 12 de 2017

Trabajo aceptado: noviembre 02 de 2017 